\title{
émulations
}

Luis Martinez, Andrade

2018

\section{Ana Cecilia Dinerstein (dir.) \\ - Social Sciences for an Other \\ Politics. Women Theorizing \\ Without Parachutes}

\section{Avertissement}

Cet article est mise à disposition selon les termes de la Licence Creative Commons Attribution - Pas d'Utilisation Commerciale 4.0 International. http://creativecommons.org/licenses/by-nc/4.0/

\section{Référence électronique}

Citation : Luis Martínez Andrade, «Ana Cecilia Dinerstein (dir.) - Social Sciences for an Other Politics. Women Theorizing Without Parachutes », Émulations, Mise en ligne le 7 juin 2018.

URL : https://ojs.uclouvain.be/index.php/emulations/article/view/ DOI :

Éditeur : Émulations - Revue de sciences sociales

Presses universitaires de Louvain

https://ojs.uclouvain.be/index.php/emulations

UCL PRESSES

UNIVERSITAIRES

DE LOUVAIN 


\title{
Ana Cecilia Dinerstein (dir.) - Social Sciences for an Other Politics. Women Theorizing Without Parachutes
}

\author{
Luis Martínez Andrade
}

Recensé : Laetitia Gérard, Le doctorat : un rite de passage. Analyse du parcours doctoral et post-doctoral, Paris, Téraèdre (« L'anthropologie au coin de la rue »), préface de David Le Breton, 2014, $211 \mathrm{p}$.

S'inscrivant dans le courant de la pensée critique contemporaine, des théoriciennes féministes nous offrent dans ce livre aussi bien des propositions théoriques stimulantes que des questions ethico-politiques pertinemment abordées à partir d'une phénoménologie de la préfiguration. Ana Cecilia Dinerstein mentionne dès l'introduction que ce « livre tente de contribuer à l'essor des formes de théorisation anti-oppressives, engagées, critiques, ouvertes et décoloniales en tant qu'outils contre le capital, le patriarcat et la colonialité ». Nous sommes donc face à un travail qui prend au sérieux les fissures (cracks) du système, c'est-à-dire le désir de révolte, l'élan de l'espérance - au sens blochien du terme - des opprimés et opprimées afin de contrecarrer la dynamique destructrice du capital et la logique homogénéisante de la modernité euro-centrée. Composé de trois volets, cet ouvrage collectif est un essai de théorisation sur le rôle des sciences sociales dans la configuration d'une autre politique.

Dans Ouvertures épistémologiques, la première partie du livre, nous observons les jalons d'une théorie critique radicale qui ne se borne plus à la réflexion de la praxis négative (Adorno, 2003) mais qui prend position pour l'affirmation de la vie. En renvoyant dos à dos le positivisme et le postmodernisme au profit d'une approche radicalement critique, cette proposition, à la fois théorique et politique, participe à l'élaboration d'une théorie critique préfiguratrice. À l'instar du penseur marxiste Ernst Bloch (1977), Sarah Amsler, Sara Motta et Dinerstein réhabilitent, chacune à leur manière, l'importance de l'utopie comme élément fondamental de la praxis, non seulement au niveau politique (luttes populaires, grèves des travailleurs, mouvements sociaux, etc.) mais aussi au niveau épistémique, à travers les concepts d' "épistémologie de la possibilité » (p. 28), de « construction dialogique » (p. 41) ou de « subsomption par exclusion» (p. 58).

1 Docteur en sociologie de l'École des hautes études en sciences sociales. Chercheur postdoctoral au Collège d'études mondiales, Paris. 
Tandis qu'Amsler s'intéresse à la question de savoir de quelle façon les « fronts ${ }^{2}$ » peuvent participer au processus d'éducation anticapitaliste et anticoloniale, Motta nous propose quant à elle de laisser de côté la figure du " prophète » (représentée à ses yeux par Slavoj Žižek ${ }^{3}$ ) et de prendre au sérieux celle du « storyteller » afin de décoloniser la pratique de la critique révolutionnaire. En s'appuyant sur les travaux de Maria Lugones, Bell Hooks et Gloria Anzaldúa, Motta appelle de ses vœux une décolonisation du savoir à partir des marges. Alors que les conséquences politiques de la pensée de Žižek ont déjà été critiquées par certains intellectuels (Castro-Gómez, 2015), selon nous, en rejetant la notion de "prophète ", Motta perd de vue l'essentiel. En effet, le prophète n'est pas celui qui connaît le futur ou prédit l'avenir, mais plutôt celui qui attire notre attention sur les menaces présentes. En ce sens, il y a eu des prophètes du $\mathrm{XX}^{\mathrm{e}}$ siècle (Simone Weil, Walter Benjamin ou Joseph Comblin, pour ne citer que quelques noms) qui peuvent encore nous aider à mettre en question les fondements de la modernité et de la colonialité réellement existantes. De son côté, Dinerstein réalise une lecture, à la lumière de l'espérance blochienne, de la critique de l'économie politique de Marx visant une décolonisation de la notion d'utopie concrète. Ce faisant, Dinerstein propose la notion de "subsomption par exclusion » (p. 58) pour désigner d'autres formes de (non) subordination des peuples indigènes dans le capital. Autrement dit, même si le capital tend à aliéner, voire à détruire, les cosmologies des peuples et des communautés, leurs résistances se trouvent toujours également en son cœur, de sorte que, là où il y a le capital, il y a de fait des luttes et des révoltes.

La deuxième partie de l'ouvrage, intitulée La (re)production de la vie, porte sur l'analyse des fissures ( cracks») dans la formation sociale hégémonique. Suzanne Bergeron aborde les contradictions qui se trouvent dans le discours des smart economics ainsi bien que dans la perspective d'analyse de rentabilisation (Business Case Approach). Selon elle, même si la logique marchande, par le biais des institutions telles que la Banque mondiale ou le Fonds monétaire international, tente d'aliéner les pratiques sociales, les

2 « Le front n'est pas un lieu physique, mais une coordonnée contingente pour la situation toujours-possible mais pas-toujours-actualisée mais dans laquelle les paramètres établis de possibilité sont déstabilisés de telle sorte que nous pouvons travailler avec le "matériel indécis" du présent » (p. 26). Amsler n'a pas tort lors qu'elle souligne que, dans l'œuvre de Bloch, il n'y pas une définition achevée de la notion de « front ».

3 Pour un dialogue critique avec la philosophie de ce penseur slovène, voir le travail de Castro-Gómez (2015) qui tente de mettre en lumière les thématiques suivantes : (1) la dimension ontologique de l'antagonisme, (2) le statut négatif de la liberté, (3) le lien entre la politique et l'universalité, (4) le caractère « incomplet » du sujet et (5) l'importance des luttes démocratiques. Plus qu'une introduction à la pensée de Žižek, le livre de Castro-Gómez dresse une cartographie de sa pensée qui démontre, à partir d'une lecture gramscienne de Nietzsche et de la théorie décoloniale latino-américaine, les contributions aussi bien que les faiblesses de la philosophie politique du philosophe slovène. 
dimensions du soin (care), de la solidarité et de la coopération sont toujours présentes et, en conséquence, participent de la configuration des possibilités. De leur côté, Raquel Gutiérrez, Lucia Linsalata et Mina Lorena Navarro soutiennent la thèse selon laquelle " la production du commun en communauté [production of the commons in common] est surtout une relation sociale en association et en coopération qui est capable de permettre quotidiennement la production sociale et la jouissance de la richesse concrète comme des valeurs d'usage, c'est-à-dire comme des biens matériels et immatériels nécessaires à la décence, à la reproduction de la vie » (p. 85). En mobilisant la pensée de Bolívar Echeverría, de Massimo de Angelis et de Silvia Federici, ces sociologues mettent en question la notion du commun (the common) proposé par Michael Hardt et Antonio Negri (2012). Selon elles, ces derniers semblent ignorer le travail reproductif et la multiplicité des relations de coopération qui se produisent à partir d'en bas et des marges. D’où l'importance de réfléchir sur les tissus communautaires ${ }^{4}$ polymorphes (polymorphic communitarian weavings). Pour sa part, Francesca Zunino Harper livre une proposition dans laquelle la notion de "natureculture » se voit comme un outil théorique et pratique pour l'analyse transdisciplinaire de la complexité sociale, culturelle et environnementale. Telle est donc la proposition faite par Harper : la mise en pratique d'une forme holistique des savoirs. On regrette un peu que Harper valorise davantage la pensée de la différence impériale (Bruno Latour, John M. Moore et Arne Næss) que celle de la différence coloniale ${ }^{5}$; il aurait été intéressant d'aborder le travail des théologiens (Boff, 1997) et des théologiennes féministes de la libération (Guevara, 1999) qui, depuis des décennies, s'intéressent à cette question.

Pour finir, Mouvements sociaux et politique préfiguratrice, la dernière partie de l'ouvrage, met en lumière les limites d'une certaine théorie des mouvements sociaux (animée principalement par Charles Tilly, Sidney Tarrow et Doug McAdam) pour comprendre la dimension préfiguratrice et subjective, ainsi que le potentiel transformateur des acteurs. Dans sa contribution, la sociologue Emily Brissette affirme qu'il est nécessaire, d'une part, d'élargir et d'enrichir ce que recouvre la notion de politique et, d'autre part, de nuancer celle de l'État. Selon elle, nous devons comprendre l'État comme une construction sociale et, pour cette raison, il ne doit pas être séparé de la société. En ce sens, la pratique politique ne doit pas seulement se limiter à rester dans les bornes de l'État. Marianne Maeckelbergh souligne quant à elle le fait que le paradigme succès/échec (success/failure paradigm) est très problématique parce qu'il s'inscrit dans la

4 Sur la notion de «tissus communautaires » voir l'excellent entretien réalisé avec Raquel Gutiérrez par Élisabeth Lagasse (Gutiérrez, Lagasse, 2016).

5 En ce qui concerne la distinction entre différence impériale et différence coloniale, se reporter à Mignolo (2012). 
logique réifiante de la doxa et du logos hégémonique. En prenant le mouvement altermondialiste ${ }^{6}$ comme cas de figure, Maeckelbergh pointe du doigt les multiples formes antihiérarchiques de l'organisation sociale, politique et économique qui façonnent les principaux traits des mouvements sociaux. De surcroît, Maeckelbergh souligne le fait que la question de la temporalité devient fondamentale pour appréhender la préfiguration. Par ailleurs, dans son excellente contribution, la sociologue Marina Sitrin nous propose de repenser les mouvements sociaux avec les « sociétés en mouvement » (Zibechi, 2014) car, à ses yeux, la perspective de la politique contestataire (Contentious Politics framework) chère à Charles Tilly et à Sidney Tarrow s'avère insuffisante pour prendre à bras-le-corps les spécificités culturelles (autonomie) ainsi bien que les traits particuliers (horizontalidad) des mouvements du Sud Global - en l'occurrence le zapatisme du sud-est mexicain. Force est de reconnaître que, dans le mouvement zapatiste, la « dignité » n'est pas seulement une catégorie éthique, elle aussi une catégorique politique, en ce sens, la « rage digne » (la digna rabia) des sociétés et communautés en mouvements devient un élan pour défier aussi bien le capitalisme que la modernité et la colonialité réellement existantes. Il est intéressant de mentionner qu'une grande partie de ces contributions établit un dialogue avec les réflexions de John Holloway (2010), penseur libertaire de l'Université autonome de Puebla (Mexique), qui depuis des années tente de produire une théorique critique ouverte, radicale et, ce faisant, une théorie d'insubordination.

Selon nous, il y a un élément qui mérite d'être approfondi dans le processus d'insubordination et de libération propre aux luttes populaires : celui de la violence. Celle non seulement du capital (abordée par Dinerstein, Gutiérrez, Linsalata et Navarro) mais aussi des opprimés et opprimées : « la violence divine »(Benjamin, 1971). Il ne faut pas oublier que le mouvement zapatiste est une armée (l’Armée zapatiste de libération nationale) qui n'a pas renoncé à la critique des armes et aux armes de la critique. Cela vaut aussi par la lutte des combattantes et combattants kurdes pour la liberté du Parti des travailleurs du Kurdistan à Rojava (Dirik, 2017) qui, les armes à la main, participent à l'actuelle reconfiguration géopolitique à l'œuvre dans la région. Sans faire une apologie de la violence, nous pensons que cet élément ne doit pas être laissé de côté si nous voulons saisir toutes les expressions de la " préfiguration politique d'en bas » auquel cet ouvrage collectif est consacré. Quoi qu'il en soit, à nos yeux, l'importante portée de ce livre réside dans le fait qu'il nous montre une autre façon de mobiliser les sciences sociales en vue de produire une autre politique. Sans renoncer à la rigueur intellectuelle, ces théoriciennes féministes ont ouvert une brèche pour l'analyse du nouveau sujet radical (new radical suject) qui est en train d'émerger, certes dans des conditions toujours adverses, mais guidé par la boussole du «principe d'espérance » (Bloch, 1977).

6 À ce sujet, voir l'excellent ouvrage de Geoffrey Pleyers (2010) dans lequel il montre les tensions autant que les convergences entre la voie de la raison et la voie de la subjectivité dans le mouvement altermondialiste. 


\section{Bibliographie}

Adorno T. (2003), Dialectique négative, Paris, Payot.

BENJAMIN W. (1971), Mythe et violence, Paris, Denoël.

Bloch E. (1977), Le Principe Espérance, Paris, Gallimard.

BofF L. (1997), Cry of the Earth, Cry of the Poor, New York, Orbis Books.

CaStro-Gomez S. (2015), Revoluciones sin sujeto. Slavoj Žižek y la crítica del historicismo posmoderno, Madrid, Akal.

DIRIK D. (2017), " Féminisme et mouvement kurde de libération », in S. BouQuiN, M. COURT, C. Hond (dir.), La commune du Rojava. L'alternative kurde à l'État-nation. Paris/Bruxelles, Syllepse/Critica, p. 147-154.

GueVARA I. (1999), Longing for Running Water: Ecofeminism and Liberation, Minneapolis, Fortress Press.

GutiÉRREZ Aguilar R., LAgasSE É. (2016), « Comprendre les luttes sociales dans et depuis le Sud Global. Entretien avec Raquel Gutiérrez », Émulations, $\mathrm{n}^{\circ}$ 19. En ligne, consulté le 14 avril 2018. URL : http://www.revue-emulations.net/archives/19perspectives-on-social-movements-from-south/comprendre-les-luttes-socialesdans-et-depuis-le-sud-global--entretien-avec-raquel-gutierrez-aguilar.

Hardt M., Negri A. (2012), Commonwealth, Paris, Stock.

Holloway J. (2010), Crack capitalism, Londres, Pluto Press.

Mignolo W. (2012), Local Histories/Global Designs: Coloniality, Subaltern Knowledges and Border Thinking, Princeton, Princeton University Press.

Pleyers G. (2010), Alter-globalization. Becoming actors in the Global Age, Cambridge, Polity.

ZiBECHI R. (2014), Descolonizar la rebeldía. (Des)colonialismo del pensamiento crítico y de las practicas emancipatorias, Malaga, Baladre. 